\title{
Removal of toluene diamine and its derivatives from polyurethane foam using immobilized Trametes versicolor
}

\author{
1,2 Åke Stenholm, 1,3 Mikael Hedeland, 1,4 Torbjörn Arvidsson and ${ }^{1}$ Curt E. Pettersson \\ ${ }^{1}$ Department of Medicinal Chemistry, Uppsala University, BMC Box 574, SE-751 23, Uppsala, Sweden \\ ${ }^{2}$ GE Healthcare Bio-Sciences AB, Björkgatan 30, SE-751 84 Uppsala, Sweden \\ ${ }^{3}$ National Veterinary Institute (SVA), Department of Chemistry, Environment and Feed, SE-751 89, Uppsala, Sweden \\ ${ }^{4}$ Medical Products Agency, Box 26, SE-751 03, Uppsala, Sweden
}

Correspondence Author: Åke Stenholm, Department of Medicinal Chemistry, Uppsala University, BMC Box 574, SE-751 23, Uppsala, Sweden ${ }^{2} \mathrm{GE}$ Healthcare Bio-Sciences AB, Björkgatan 30, SE-751 84 Uppsala, Sweden

E-mail: ake.stenholm@ge.com

Received date: 12 December 2018, Accepted date: 13 February 2019, Online date: 25 February 2019

Copyright: (c) 2019 Åke Stenholm et al., This is an open-access article distributed under the terms of the Creative Commons Attribution License, which permits unrestricted use, distribution, and reproduction in any medium, provided the original author and source are credited.

\begin{abstract}
Background: In addition to uses in home furnishings and the construction sector, polyurethane foam (PUF) is also prevalent in medical products such as scaffolds and implants. However, these applications raise concerns for human health as the use of this material can result in exposure to the carcinogenic substance 2,4-toluenediamine (2,4-TDA). The objective of this study was to identify easily extracted PUF-residuals and to study the removal of them in a biodegradation experiment including immobilized Trametes versicolor. Ultra-high-performance liquid chromatography-quadrupole/time of flight mass spectrometry (UHPLC-Q-TOF MS) was used in MS and MS/MS-mode to confirm the identities of PUF-related compounds and follow their concentration changes. Results: Except for 2,4-TDA and 2,6-TDA which were confirmed by standards, previously not reported substances were tentatively identified, among them TDA-dimers. These dimers include homodimers, heterodimers containing 2,4-TDA and 2,6-TDA and compounds that are hydroxylated. The experiments that were performed in an acidic fungal culture revealed a not previously described removal of these compounds below the approximate nM detection limit. The mechanisms behind their removal may include biosorption to fungal mycelia, bioaccumulation, use of them as nutrients or extracellular catabolism. Conclusion: The results of this study not only highlight the ease by which harmful compounds were extracted from the investigated PUF-quality which is used in non-medical applications, but also the possibility to use fungal-based methods to eliminate them. This could be facilitated by an initial extraction of PUF (excluding fungi) followed by a removal of the substances with PUF-immobilized Trametes versicolor. The findings in this study may be of interest to further investigate PUF-residuals in products aimed for medical applications.
\end{abstract}

Key words: 2,4-toluenediamine, Trametes versicolor, removal, liquid chromatography, mass spectrometry.

\section{INTRODUCTION}

Polyurethane (PU) is used in numerous medical applications including catheter and general purpose tubings, surgical drapes and wound dressings. One other common use is in implants. It is an abundant plastic that is composed of urethane linkages. It is formed by reacting a di- or polyisocyanate with a polyol through an exothermic polymerization process (see Fig. 1).

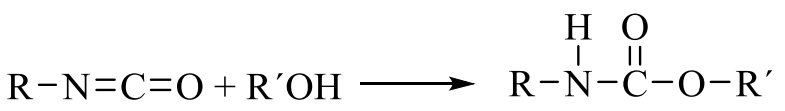

Fig. 1: General urethane condensation reaction

The most commonly used isocyanates are toluene diisocyanate (TDI) and methylene bisphenyl diisocyanate (MDI). TDI is often an 80:20 mixture of 2,4-toluene diisocyanate (2,4-TDI) and 2,6-toluene diisocyanate (2,6-TDI). 2,4-TDI is highly toxic. The main commercial polyols that are used to make PU are polyether- or polyester-based. The ether and ester bonds have lower hydrolytic stability than the urethane bond [1]. Pavlova et al. determined the hydrolytic stability of polyurethanes based on 
polyester and polyether polyols and concluded that the hydrolytic stability of polyether polyols was higher in alkaline (20\% $\mathrm{NaOH})$ and aqueous media while polyester polyols were more stable in acidic medium $\left(20 \% \mathrm{HNO}_{3}\right)$ [2]. The hydrolysis of urethane bonds requires extreme conditions, such as temperatures over $100{ }^{\circ} \mathrm{C}$ or highly alkaline or acidic conditions.

Furthermore, most important, hydrolysis of polyether or polyester polyols in polyurethane cannot produce TDA since the production of one molecule TDA requires the rupture of two urethane bonds [3].

Polyurethane foam (PUF) is produced by adding a blowing agent which evaporates during the exothermic reaction and yields bubbles, leading to soft porous polymers [4]. It has been reported that unreacted TDI is present in PUF [5,6]. However, these results have been questioned since it was not concluded whether the detected TDI stemmed from residual TDI or from foam degradation during extraction or derivatization procedures [7].

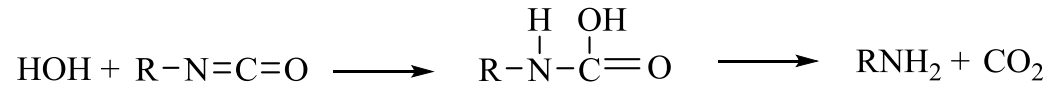

Fig. 2: Reaction between isocyanate and water

Figure 2 shows the conversion of an isocyanate to a primary amine. Thus, the residual 2,4-TDI and 2,6-TDI molecules in PUF are transformed to 2,4-toluene diamine (2,4-TDA) and 2,6-toluene diamine (2,6-TDA), respectively, upon contact with water.

2,4-TDA is a class A carcinogen [8]. Experiments in rats demonstrated that the hydroxylamine resulting from 2,4-TDA hydroxylation by hepatic microsomes is genotoxic based on a positive response in the Ames test. The hydroxylamine was shown to promote the cellular proliferation of mutated cells. In contrast to 2,4-TDA, 2,6-TDA is a mutagen rather than a carcinogen [9].<smiles>Cc1ccc(N)cc1N</smiles><smiles>Cc1c(N)cccc1N</smiles>

(B)

Fig. 3: Chemical structures of 2,4-TDA (A) and 2,6-TDA (B).

When this is taken into account, in vivo PU/PUF applications must be carefully considered. One of these is medical uses including breast silicone gel implant covers. The leakage of 2,4-TDA from polyester-based PUF implant covers has been investigated in both a human trial and a rat study. In the study performed on women, approximately half (30 out of 61) of patients had measurable levels of 2,4-TDA in their urine [10]. In the study performed on female rats [11], a low release of 2,4-TDA was monitored in the plasma samples. This research concluded that polyurethane foam-covered breast implants present an unreasonable health risk when the results were extrapolated to reflect 2,4-TDA bioavailability in humans.

The in vivo release of 2,4-TDA from PUF is influenced by several factors that act synergistically. These are (1) enzymatic activities, (2) oxidizing agents, and (3) mechanical loads (environmental stress cracking). The enzymatic degradation not only includes the hydrolysis of polyester and polyether, but also the cleavage of polyurethane bonds [12]. Thus, the in vivo release of 2,4-TDA from PUF following the cleavage of urethane bonds can occur at mild conditions with respect to $\mathrm{pH}$ and temperature.

The extraction conditions are vital to extracting residual TDA from a PUF sample. That is, the residuals must be readily soluble yet the polyester or polyether groups in the PU-oligomers cannot be allowed to hydrolyze in solution. This hydrolysis of polyester and polyether groups could cause an amalgam of acids and alcohols that, in the worst case, would render the reliable quantification of residual TDA impossible.

Fortunately, 2,4-TDA and 2,6-TDA can be easily extracted from PUF. Johnson et al. [13] used a $0.1 \%$ w/v acetic acid solution $(\mathrm{pH}=3.3)$ to extract these compounds. The $\mathrm{pKa}$ values for the conjugate-acid forms of 2,4-TDA and 2,6-TDA have been reported as 5.58 and 4.69, respectively [14]. In the study performed by Johnson et al., the protonated amines were analyzed using hydrophilic interaction chromatography (HILIC) and tandem mass spectrometry (MS/MS) employing positive electrospray ionization. A similar extraction protocol (i.e. under acidic conditions) was applied by Mutsuga et al. [15].

Earlier studies have questioned whether the TDA detected in PUF samples is a hazard or an analytical artefact (a similar issue exists for TDI and PUF) [7]. Hall et al. [16] draw the conclusion that the urethane oligomers in PUF extracts containing $10^{-4} \mathrm{M}$ $\mathrm{NaOH}$ could hydrolyze into TDA. However, this study, which used GC-MS, did not consider the risk of thermal degradation of these oligomers when passing through the heated liner. It has been shown that the depolymerization of polyurethanes starts at temperatures $<280{ }^{\circ} \mathrm{C}$ [17], and Hall et al. used an injector temperature of $250{ }^{\circ} \mathrm{C}$.

Advanced oxidation procedures (AOPs) have been used to degrade 2,4-TDA in synthetic wastewaters [18]. The results showed that this amine species, initially at a concentration of $0.5 \mathrm{mM}\left(61.0 \mathrm{mg} \mathrm{L}^{-1}\right)$, could be completely removed from water at $\mathrm{pH} 7$ by treatment with $\mathrm{UV} / \mathrm{H}_{2} \mathrm{O}_{2}$. Successful biodegradation of TDA-isomers has been performed in activated sludge acclimated with aniline and TDA [19]. The acclimation period was approximately 200 days and it was determined that no external carbon sources were needed for the biodegradation. Another study explored selected bacteria and fungi for the biodegradation of TDA 
isomers [20]. The TDA isomers were the sole carbon source, and Thielevia sp. (phylum Ascomycota) was chosen for degrading 2,4-TDA. The results showed that the fungus was faster at degrading this particular diamine than the bacteria.

The objective of the present study was to identify easily extracted PUF-residuals and to study the removal of them in a biodegradation experiment including immobilized Trametes versicolor. In the present investigation, PUF cubes were used as supports (carriers) for immobilized Trametes versicolor (T. versicolor) mycelia. The choice of this white rot fungus (WRF) species was motivated by its reported use in the biodegradation of small aromatic xenobiotic compounds [21,22]. The ability of immobilized $T$. versicolor to remove 2,4-TDA and 2,6-TDA, as well as their derivatives, from PUF was studied in a nutrient solution ( $\mathrm{pH} 4)$.

The mild extraction conditions excluded any hydrolysis of polyurethane and should thus only include residuals of TDA and its derivatives. The identification of these compounds was performed using both electrospray ionization (ESI) UHPLC-Q-TOF-MS and MS/MS in positive mode. The presented removal procedure is novel. The findings can be useful for PUF applications which do not allow any leachage of residual substances.

\section{MATERIALS AND METHODS}

\section{Chemicals and glassware}

PUF (Eheim pickup 160) was purchased from Eheim GmbH \& Co. KG (Deizisau, Germany). This PUF quality is used in filters for home aquariums. Diclofenac sodium salt, D-(+)-glucose ( $\geq 99.5 \%)$, ammonium tartrate dibasic ( $\geq 98 \%$ ), 2,4diaminotoluene (98\%) and 2,6-diaminotoluene (97\%) were purchased from Sigma-Aldrich (St. Louis, MO). Diclofenac-d4 with an isotopic enrichment of 98 atom\% deuterium, was obtained from C/D/N Isotopes Inc. (Quebec, Canada). Emsure® Sulfuric acid (95-97\%), LiChrosolv ${ }^{\circledR}$ Hypergrade acetonitrile $(\geq 99.9 \%)$, and LiChrosolv ${ }^{\circledR}$ water for chromatography were purchased from Merck (Darmstadt, Germany). Ammonium acetate $(\geq 99.0 \%)$ for the preparation of the mobile phase was obtained from Fluka (Darmstadt, Germany). The Erlenmeyer borosilicate glass flasks (VWR International, Radnor, PA) included polypropylene (PP) screw-caps and polytetrafluoroethylene (PTFE) membranes through which air can diffuse. The nutrient solution used throughout the experiments (including fungal growth onto immobilization supports and biodegradation experiments) contained $6.0 \mathrm{~g} \mathrm{~L} \mathrm{~L}^{-1} \mathrm{D}$ (+)-glucose and $3.3 \mathrm{~g} \mathrm{~L}^{-1}$ ammonium tartrate with a carbon: nitrogen molar ratio $(\mathrm{C}: \mathrm{N})$ of 6.6 . The $\mathrm{pH}$ of the solution was adjusted to 4.0 with $1.0 \mathrm{M}$ sulfuric acid.

Tap water from General Electric Healthcare (softened at site), Uppsala, Sweden was used unless otherwise specified. Fungus:

T. versicolor (strain AG1383) was obtained from the Culture Collection of Basidiomycetes, Institute of Microbiology, Academy of Sciences of the Czech Republic in Prague. The fungus was maintained by subculturing every second month onto disks containing modified malt extract agar supplied by the National Veterinary Institute (SVA, Uppsala, Sweden) at ambient temperature. At each subculturing event, an aseptic (70\% ethanol) hollow punch with an inner diameter of $10 \mathrm{~mm}$ was used to sample a mycelia-covered disk and then transfer the sample to a new plate (one agar pellet for each new plate). After one week of storage in the dark at ambient temperature, the prepared disks were stored in a refrigerator for a maximum time period of two months.

\section{Instrumentation and general MS-settings}

An Agilent Technologies 6550 iFunnel Q-TOF LC/MS system (Agilent Technologies, Santa Clara, CA), including an Agilent Technologies 1290 Infinity UHPLC system consisting of a 1260 iso pump (G1310B), 1290 binary pump (G4220A), thermostat (G1330B), 1290 sampler (G4226A), 1290 thermostated column compartment (G1316C) and electrospray ionization (ESI) was used for quantitative analysis. Agilent MassHunter software (version 06.00) was used for data acquisition and processing. The drying gas flow rate and temperature were set to $14 \mathrm{~L} \mathrm{~min}^{-1}$ and $150{ }^{\circ} \mathrm{C}$, respectively, while the sheath gas flow and temperature were set to $11 \mathrm{~L} \mathrm{~min}^{-1}$ and $350{ }^{\circ} \mathrm{C}$, respectively. The capillary voltage was set at $4000 \mathrm{~V}$. The mass spectrometer was operated in a

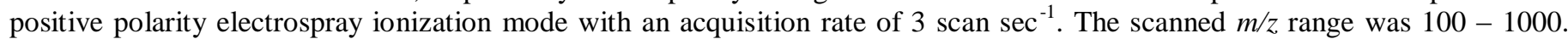
Lock masses with $m / z 121.05087300$ (protonated purine) and $m / z 922.00979800$ (protonated hexakis- $(1 \mathrm{H}, 1 \mathrm{H}, 3 \mathrm{H}-$ tetrafluoropentoxy) phosphazene) were used. At each analysis occasion (i.e. day), the instrument was calibrated in positive mode using the Agilent CheckTune procedure, which demands a mass accuracy $<1 \mathrm{ppm}$ and a resolution $>20000$. A Zorbax Eclipse plus $\mathrm{C}_{18}$ rapid resolution HD $(2.1 \times 50 \mathrm{~mm}, 1.8 \mu \mathrm{m})$ UHPLC-column from Agilent Technologies was used for separation purposes.

\section{Experiments in Erlenmeyer flasks containing PUF-carriers:}

The experiments were performed in $500 \mathrm{~mL}$ bottom-baffled Erlenmeyer flasks. Three flasks were filled with $300 \mathrm{~mL}$ nutrient solution each. Approximately $2.7 \mathrm{~g}$ of PUF cubes $\left(1 \mathrm{~cm}^{3}\right)$ were added to each of the three Erlenmeyer flasks, after which the flasks were autoclaved $\left(125^{\circ} \mathrm{C}, 30\right.$ minutes). The autoclavation procedure was motivated by the desire to create an environment, initially free from bacterial contamination. Two of the flasks were inoculated with 15 agar plugs covered with mycelia. All of the flasks were agitated $(50 \mathrm{rpm})$ for one week, after which one of the flasks containing mycelia was withdrawn and autoclaved to establish a heat-killed control. Thus, the three flasks contained three different conditions: (1) active fungus; (2) heat-killed control and (3) carrier control. Diclofenac was then added to the flasks to a final concentration of $10 \mathrm{mg} \mathrm{L}^{-1}$. The removal of this compound by adsorption and T. versicolor mediated biodegradation mechanisms is previously reported [22]. Nutrient stock solution stored in a $1000 \mathrm{~mL}$ glass bottle was used as the blank in UHPLC-Q-TOF MS runs. Samples, $4 \mathrm{~mL}$ each, were taken from the three flasks at $0,1,2,4,6,22,29,46,70,94$ and 166 hours. From the collected samples, $2.2 \mathrm{~mL}$ was used for a laccase enzyme assay and $1.4 \mathrm{~mL}$ for UHPLC-Q-TOF analysis. The latter samples were frozen to stop the secretion of enzymes. The time between sampling and a sample entering the freezer was estimated to be 15 minutes. For subsequent experiments, an internal 
standard (diclofenac-d4) was added to a concentration of $0.1 \mathrm{mg} \mathrm{L}^{-1}$ once the samples had thawed, after which the samples were centrifuged and transferred to glass vials. The diclofenac-d4 was initially added for the purpose of measuring diclofenac decay [22]. In the present study, it was used to semi-quantitatively determine the concentration levels of TDA and its derivatives. The UHPLC-QTOF MS analyses were performed in triplicates.

\section{UHPLC-Q-TOF MS and MS/MS-analyses}

Mobile phases A and B consisted of LiChrosolv ${ }^{\circledR}$ water containing $2.0 \mathrm{mM}$ ammoniumacetate (pH 7.0) and hypergrade acetonitrile, respectively. The flow rate and temperature were set to $0.3 \mathrm{~mL} \mathrm{~min}^{-1}$ and $30{ }^{\circ} \mathrm{C}$, respectively. The mobile phase contained initially $90 \%$ A. After a 6 minutes linear decrease to $10 \%$ A, an isocratic period of one minute followed, after which this phase was linearly increased from $10 \%$ to $90 \%$ over a time period of one minute and then kept at this concentration for three minutes to equilibrate the column. Next, $3 \mu \mathrm{L}$ of undiluted sample was injected. MS data were obtained by positive ESI. Eluted peaks were analyzed in Q-TOF full-scan MS mode, MS/MS mode and by measuring absorbance at $276 \mathrm{~nm}$. Diode array spectra (DAD) were continuously collected over the $100-600 \mathrm{~nm}$ range. The MS/MS fragmentations were performed using three collision energies (10, 20 and $40 \mathrm{~V}$ ). The collision gas of choice was Nitrogen 5.0 with a purity of $99.99999 \%$ from AGA (Lidingö, Sweden), and the collision pressure was set to 2.1 bar. In the full-scan mode, accurate protonated masses were compared with exact masses of TDA and TDA-derivatives. An acceptable error limit of $2.5 \mathrm{ppm}$ was applied to these mass measurements. Spectra from detectable TIC-peaks were investigated, and the most abundant ions were evaluated using the ChemSpider search engine.

\section{Statistical methods:}

In Table 1, the calculations of accurate and exact masses in MS and accurate masses in MS/MS-mode, were facilitated by use of Agilent MassHunter software (version 06.00). Furthermore, using this software, elemental compositions were suggested for detected ions. In full scan MS-mode, the software ChemSpider was used to evaluate possible chemical structures of suggested elemental compositions [23]. The elemental compositions of the product ions were interpreted by the web-based program ChemCalc [24].

\section{RESULTS AND DISCUSSION}

The experimental culture (EC) included a live T. versicolor culture, nutrient solution and PUF-carriers. The heat-killed control (HKC) consisted of nutrient solution, carriers, and a T. Versicolor culture that had been killed during autoclaving. The carrier control (CC) contained only carriers and nutrient solution. Blank (BL) samples refer to samples containing only the nutrient solution.

The results of UHPLC-Q-TOF MS (full-scan mode) for samples from various experimental conditions (EC, HKC and CC), compared against a BL run, are shown in Fig. 4. The samples were collected from the Erlenmeyer flasks (EC, HKC, CC) two hours after the addition of diclofenac. Substances that were identified (TDA) and tentatively identified (TDA-derivatives) are marked with indices (1-12).

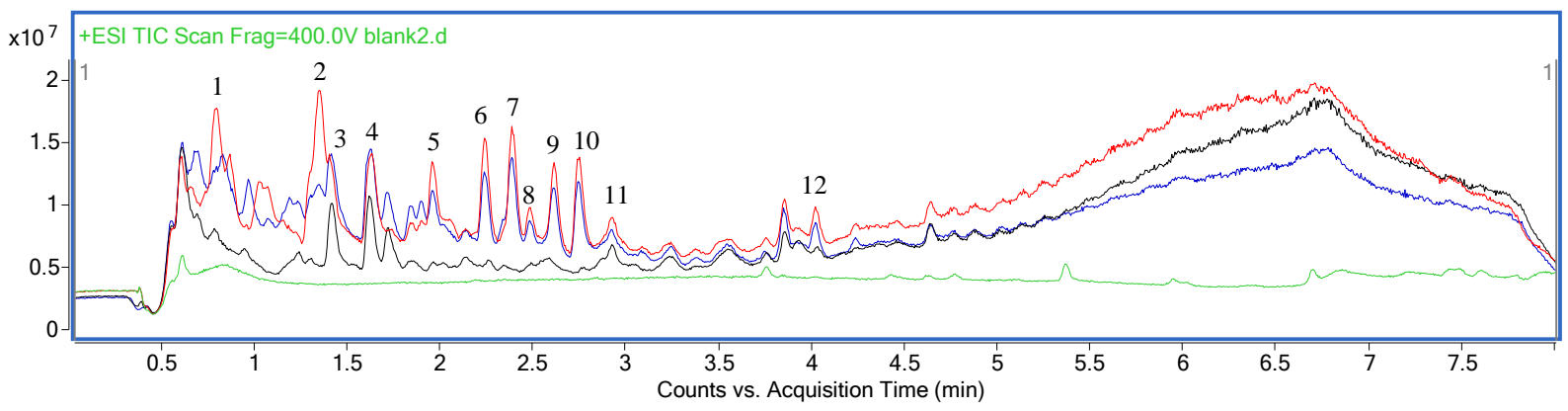

Fig. 4: UHPLC-Q-TOF MS results for samples collected two hours after the addition of diclofenac. The addition of diclofenac was preceded by one week of fungal immobilization on PUF (EC and HKC). Total Ion Chromatograms (TIC); Colors: EC (black), HKC (blue), CC (red) and (BL) green.

The data files were processed by the Find Compound by Formula algorithm and the two isomers of TDA were identified in peaks $1\left(t_{\mathrm{R}} 0.81 \mathrm{~min}\right.$.) and $2\left(t_{\mathrm{R}} 1.32 \mathrm{~min}\right.$.) of HKC and CC, but not in EC- samples by a $[\mathrm{M}+\mathrm{H}]^{+}$ion at $\mathrm{m} / z$. $123.0919($ accurate mass), which differs by $1.6 \mathrm{ppm}(0.2 \mathrm{mDa})$ from the theoretical (exact mass) $\mathrm{m} / \mathrm{z}$ of the proton adduct of TDA (123.0917). An integration of the extracted ion chromatogram (EIC) areas for peaks 1 and 2 of the CC sample showed that $m / z$, 123.0919 was over 4 times more abundant in peak 2 than in peak 1 . The identities were confirmed by injecting samples containing reference solutions of the compounds at $100 \mu \mathrm{g} \mathrm{L}^{-1}$ in nutrient solution into the chromatographic system. The retention times were 0.83 min. (peak 1 ) and 1.27 min. (peak 2) and the accurate masses were $\mathrm{m} / \mathrm{z} 123.0920$ for both reference samples. The mass error was 2.4 ppm ( 0.3 $\mathrm{mDa}$ ). Product ion scans for the precursor ion $(\mathrm{m} / \mathrm{z}$ 123.0919) in CC over retention time windows that covered peaks 1 and 2 showed similar product ions in both peaks with $\mathrm{m} / \mathrm{z} 77.0397$ and 108.0693. These ions were found at CIDs of 10, 20 and $40 \mathrm{~V}$. The mass-to-charge ratios correlate with a phenyl cation and a de-methylated cation radical of TDA, respectively. The mass errors 
were 7.4 and $8.3 \mathrm{ppm}$, respectively. A larger mass error in MS/MS is explained by the absence of lock-masses in the TOF. MS/MS was also performed on 2,4-TDA and 2,6-TDA references dissolved in nutrient solution to $100 \mu \mathrm{g} \mathrm{L}^{-1}$. Their product ions were similar to the experimental findings in CC ( $\mathrm{m} / \mathrm{z}, 77.0396,108.0691$ and 77.0398 and 108.0691 respectively). The conclusion was that peaks 1 and 2 in Fig. 4 contained 2,6-TDA and 2,4-TDA, respectively.

Full-scan spectra were collected for peaks 3 and 4. One abundant ion was present $(\mathrm{m} / \mathrm{z}$ 177.0661) in both peaks. Since the compound was present in EC, HKC and CC, it must be derived from PUF. No decline of the peak intensity was visible in any of the controls through the experiment. The ratios between the integrated ion intensities at $t_{\mathrm{R}} 1.4$ and 1.6 min. and the integrated IS (diclofenac d-4) intensity at $\mathrm{m} / \mathrm{z} 300.0496$ were approximately 8 (the IS concentration was $0.1 \mathrm{mg} \mathrm{L}^{-1}$ ). Using the MassHunter algorithm "generate formulas from spectrum", a proton adduct with an elemental composition of $\mathrm{C}_{9} \mathrm{H}_{9} \mathrm{~N}_{2} \mathrm{O}_{2}$ was suggested. The nitrogen rule confirmed the presence of two nitrogen atoms. The exact $\mathrm{m} / \mathrm{z}$ for the ion is 177.0659 , which reflects a mass error of $1.1 \mathrm{ppm}$. The MassHunter MS score for the compound was $98 \%$ using default settings for MS accuracy, isotope spacing and abundance contribution to the score. Product ion spectra were acquired and are shown in Fig. 5. In Fig. 5B which represents the 40V CID energy, a water loss is present at $\mathrm{m} / \mathrm{z}$ 159.0550. The ion at $\mathrm{m} / \mathrm{z} 131.0602$ in Figs. 5A and 5B matches the ion species $\left[\mathrm{M}+\mathrm{H}-\mathrm{CH}_{2} \mathrm{O}_{2}\right]^{+}$. The product ion at $\mathrm{m} / z, 119.0490$ which is seen in Fig. 5A, can be explained by an $\left[\mathrm{M}+\mathrm{H}-\mathrm{CH}_{2} \mathrm{~N}_{2} \mathrm{O}\right]^{+}$ion species, whereas the ion at $m / z$ 104.0490, which is abundant in Fig. $5 \mathrm{~B}$, was considered to be an $\left[\mathrm{C}_{7} \mathrm{H}_{6} \mathrm{~N}\right]^{+}$ion species $(\mathrm{mass}$ error $4.8 \mathrm{ppm})$. The ion at $\mathrm{m} / z, 91.0538$ is explained by a tropylium $\left[\mathrm{C}_{7} \mathrm{H}_{7}\right]^{+}$ion. Furthermore, three characteristic ions were abundant $(\mathrm{m} / \mathrm{z}$ values of 77.0386, 65.0380 and 51.0226) in Figure 5B: the ion at $\mathrm{m} / \mathrm{z} 77.0386$ most probably represents a phenyl cation $\left[\mathrm{C}_{6} \mathrm{H}_{5}\right]^{+}$while the other two ions originate from the fragmentation of benzene molecules into $\left[\mathrm{C}_{5} \mathrm{H}_{5}\right]^{+}\left(\mathrm{m} / z\right.$ 65.0380) and $\left[\mathrm{C}_{4} \mathrm{H}_{3}\right]^{+}$ $(\mathrm{m} / \mathrm{z}$ 51.0226) (see Fig. 5 and Table 1).
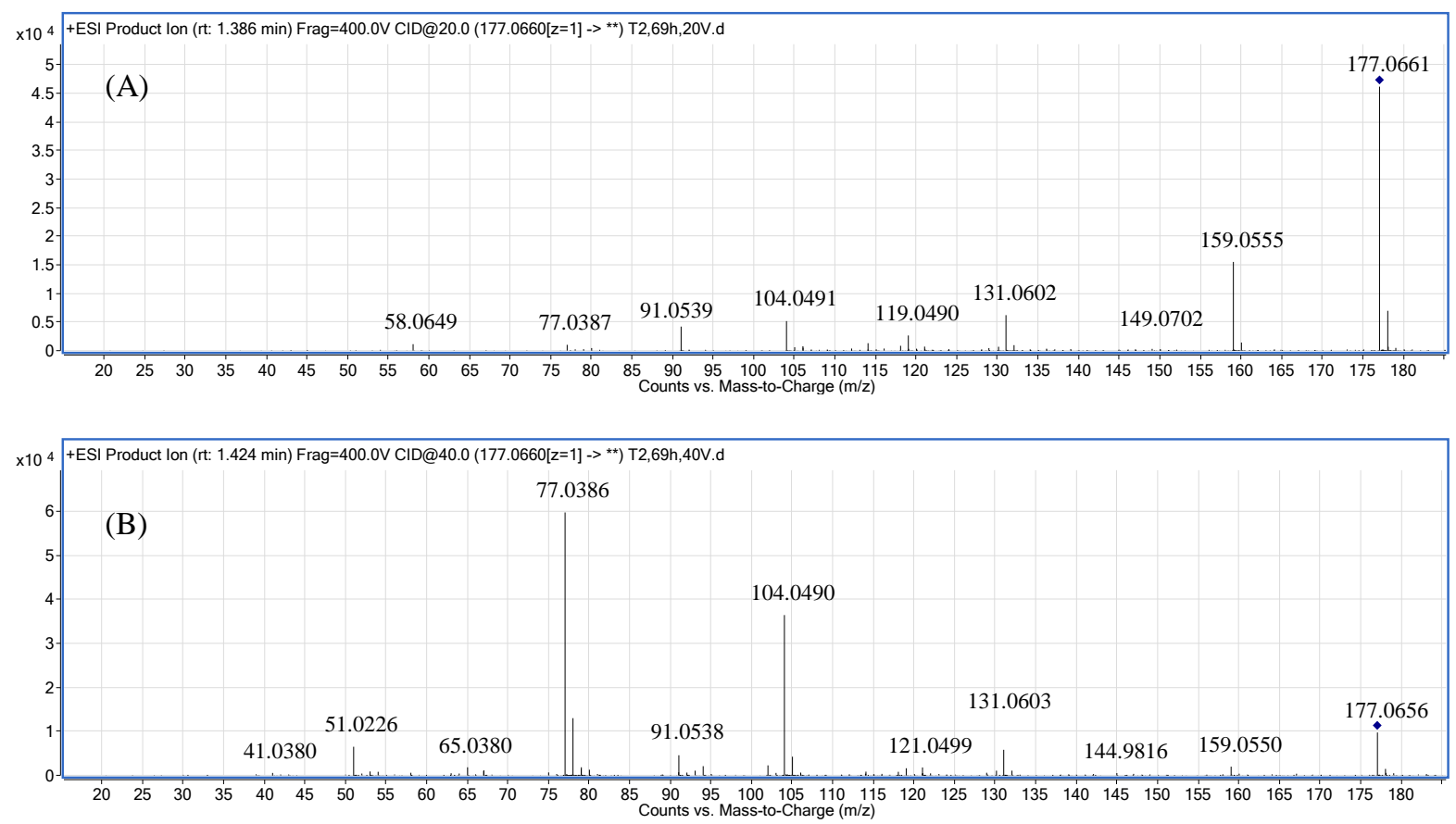

Fig. 5: Product ion spectra of a precursor ion with $\mathrm{m} / z, 177.0660$ (A), $20 \mathrm{~V}$ CID and (B), 40V CID. The analysed EC sample was collected at $69 \mathrm{~h}$.

Table 1: Summary of accurate mass measurements of fragment ions in product ion spectra of EC sample collected at $69 \mathrm{~h}$. using UHPLC-Q-TOF MS/MS. Precursor ion: $m / z$ 177.0660. The CID-values show at which collision induced energy the respective $m / z$ was obtained. The elemental compositions are shown in their uncharged state. 


\begin{tabular}{lllcclcc}
\hline $\begin{array}{l}\text { Elemental } \\
\text { composition }\end{array}$ & Accurate & mass, $\mathrm{m} / \mathrm{z}$ & \multicolumn{1}{c}{ Exact } & \multicolumn{2}{c}{ Mass error } & Ionic species & CID (V) \\
\hline $\mathrm{C}_{9} \mathrm{H}_{8} \mathrm{~N}_{2} \mathrm{O}_{2}$ & 177.0661 & 177.0659 & -0.1 & -1.1 & {$[\mathrm{M}+\mathrm{H}]^{+}$} & 10,20 \\
$\mathrm{C}_{9} \mathrm{H}_{7} \mathrm{~N}_{2} \mathrm{O}$ & 159.0550 & 159.0553 & 0.3 & 1.9 & {$\left[\mathrm{M}+\mathrm{H}-\mathrm{H}_{2} \mathrm{O}\right]^{+}$} & 40 \\
$\mathrm{C}_{8} \mathrm{H}_{7} \mathrm{~N}_{2}$ & 131.0602 & 131.0604 & 0.2 & 1.5 & {$\left[\mathrm{M}+\mathrm{H}-\mathrm{CH}_{2} \mathrm{O}_{2}\right]^{+}$} & 40 \\
$\mathrm{C}_{8} \mathrm{H}_{7} \mathrm{O}$ & 119.0490 & 119.0497 & 0.7 & 5.9 & {$\left[\mathrm{M}+\mathrm{H}-\mathrm{CH}_{2} \mathrm{~N}_{2} \mathrm{O}\right]^{+}$} & 20 \\
$\mathrm{C}_{7} \mathrm{H}_{6} \mathrm{~N}$ & 104.0490 & 104.0495 & 0.5 & 4.8 & {$\left[\mathrm{M}+\mathrm{H}-\mathrm{C}_{2} \mathrm{H}_{3} \mathrm{NO}_{2}\right]^{+}$} & 40 \\
$\mathrm{C}_{7} \mathrm{H}_{7}$ & 91.0548 & 91.0538 & -1.0 & -10.9 & {$\left[\mathrm{M}+\mathrm{H}-\mathrm{C}_{2} \mathrm{H}_{2} \mathrm{~N}_{2} \mathrm{O}_{2}\right]^{+}$} & 40 \\
$\mathrm{C}_{6} \mathrm{H}_{5}$ & 77.0391 & 77.0386 & -0.5 & -6.5 & {$\left[\mathrm{M}+\mathrm{H}-\mathrm{C}_{3} \mathrm{H}_{4} \mathrm{~N}_{2} \mathrm{O}_{2}\right]^{+}$} & 40
\end{tabular}

Based on MS/MS data and an intrinsic property search in ChemSpider Chemical Database, the compound was tentatively identified as a hetoerocyclic molecule including a toluene moiety, and in the other ring structure, two oxygen and two nitrogen atoms are present. The water loss in the product ion spectra indicates that at least one of the oxygen atoms is present as a hydroxyl. The presence of a toluene moiety and two nitrogen atoms indicate that the origin is TDI. However, the ring structure requires a molecule with the isocyanate groups in ortho-position. It is known that in the synthesis of TDI using TDA and phosgene, unwanted ortho-TDI is formed. It is also known that the two adjacent isocyanate groups react by forming tolyl imidazoline-one [25]. However, this compound has an elemental composition of $\mathrm{C}_{8} \mathrm{H}_{8} \mathrm{~N}_{2} \mathrm{O}$. Thus, $\mathrm{CO}$ is missing to match the investigated substance with the elemental composition $\mathrm{C}_{9} \mathrm{H}_{8} \mathrm{~N}_{2} \mathrm{O}_{2}$ (uncharged state). A carboxylation of tolyl imidazoline-one using $\mathrm{CO}_{2}$ that was evolved in the iscocyanate - water reaction cannot be excluded (see Fig. 2). Previously a $\mathrm{CO}_{2}$ mediated synthesis (cyclization) of quinazoline-2,4 $(1 \mathrm{H}, 3 \mathrm{H})$-dione in water was reported using 2-aminobenzonitrile and dimethyl formamide in dense $\mathrm{CO}_{2}$ [26]. In the present study, the compound, preferably contain a quinoxaline backbone. See Fig.6. The presence of two peaks ( 3 and 4 in Fig.4) is explained by alternative methyl group positions.<smiles>O=c1[nH]c2ccccc2nc1O</smiles>

Fig. 6: Chemical structure of methyl $(1 \mathrm{H})$-quinoxaline-2-one, 3-ol. 1

As previously mentioned, no decline of the compound(s) was seen during the experiment in any of the controls or in the experimental culture. This means that they adsorb poorly to PUF and biomass. Furthermore, quinoxalines are known as antifungal agents, which means that it is not expected that $T$. versicolor can use them as nutrients [27].

Full-scan spectra were collected for peaks 5-10, which were only present in HKC and CC samples (Fig. 4). Two relatively abundant ions were identified in these spectra $(\mathrm{m} / z, 271.1562$ and 287.1509). These mass-to-charge ratios correspond well with proton adducts of (1) a TDA-dimer and (2) a hydroxylated TDA-dimer (TDA-dimer-OH), respectively. The TDA-dimer formation is explained by the reaction between an isocyanate group in one TDA molecule with an amine-group in another molecule [28].

$\mathrm{R}^{\prime} \mathrm{NH}_{2}+\mathrm{R}-\mathrm{N}=\mathrm{C}=\mathrm{O} \longrightarrow \begin{array}{r}\mathrm{H} \quad \mathrm{NHR}^{\prime} \\ \mathrm{R}-\mathrm{N}-\mathrm{C}^{\prime}=\mathrm{O}\end{array}$

Fig. 7: Reaction between an isocyanate and an amine (urea formation).

The accurate mass of the TDA-dimer $[\mathrm{M}+\mathrm{H}]^{+}$ion $(\mathrm{m} / z$ 271.1562) showed a mass error of $1.1 \mathrm{ppm}(0.3 \mathrm{mDa})$ when compared to the exact mass of the ion $(\mathrm{m} / z$ 271.1559). The accurate mass of the proton adduct of the TDA-dimer-OH was $\mathrm{m} / \mathrm{z}$ 287.1509 , which shows a mass error of $0.3 \mathrm{ppm}(0.1 \mathrm{mDa})$ when compared to the exact mass of the ion $(\mathrm{m} / 2.287 .1508)$. The product ion spectra are shown in Figures 8 and 9.

The presence of peaks 5-10 in Fig.4, can be explained by different isomeric forms of TDA, including homodimers, heterodimers containing 2,4-TDA and 2,6-TDA, and compounds that are hydroxylated at various positions. Proton adducts of the TDA-dimer-OH isomers $(\mathrm{m} / z 287.1509)$ were most abundant at peaks with retention times of $2.5,2.6$ and 2.8 minutes whereas the proton adducts of the TDA-dimer isomers $(\mathrm{m} / \mathrm{z} 271.1562)$ were abundant in earlier peaks (retention times of 2.0, 2.2, and 2.4 minutes) 


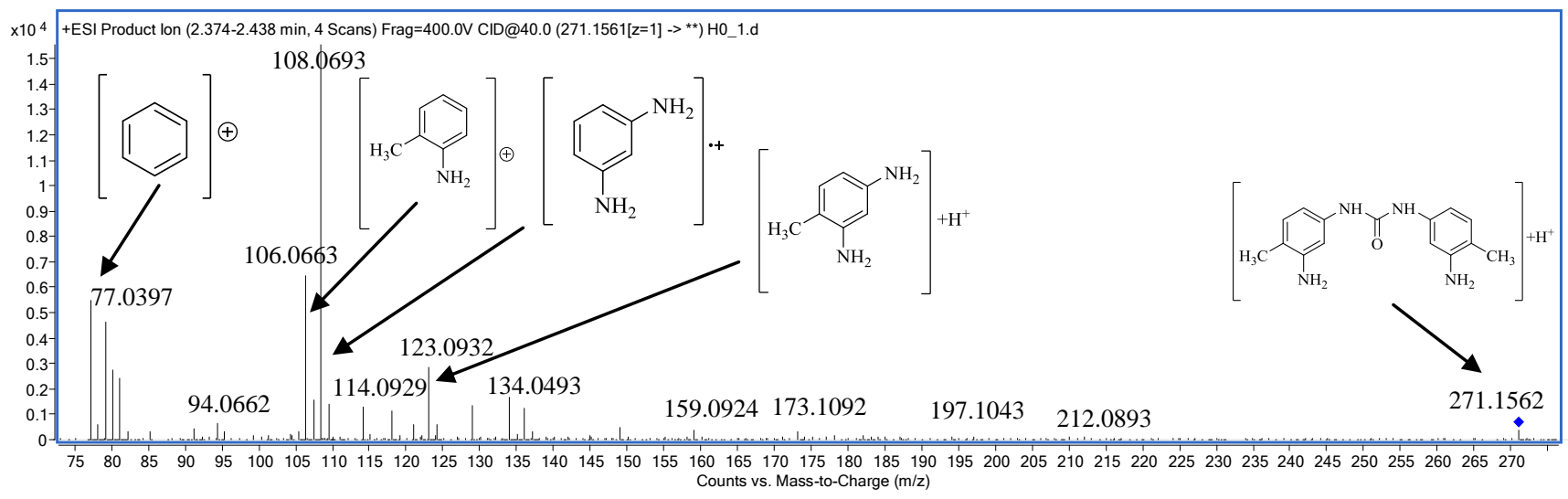

Fig. 8: Product ion spectrum of TDA-dimer isomer using a precursor ion with $\mathrm{m} / \mathrm{z} 271.1562$, with collision voltage set to $40 \mathrm{~V}$. The analyzed sample was collected from the heat-killed culture (HKC) one day after the addition of diclofenac, which was preceded by one week of fungal immobilization on PUF.

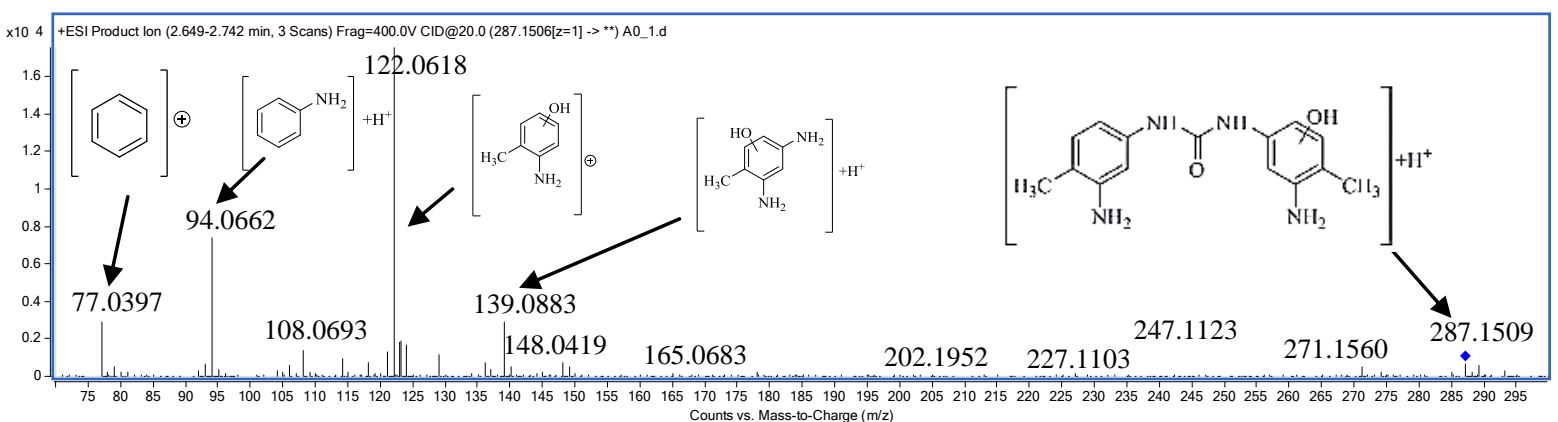

Fig. 9: Product ion spectrum of a hydroxylated TDA-dimer isomer using a precursor ion with $\mathrm{m} / \mathrm{z}$ 287.1509, with collision voltage set to $20 \mathrm{~V}$. CC-sample collected one day after the addition of diclofenac.

The mass accuracies of the product ions (including proton adducts, carbocations and cation radicals), shown in Figures 8 and 9 were all below 10 ppm. Peak 11 in Fig. 4 is identical with IS (diclofenac d-4). Peak 12 in Fig. 4 had an abundant ion at $m / z$ 249.1959. Using the MassHunter algorithm "generate formulas from spectrum", a proton adduct with an elemental composition of $\mathrm{C}_{15} \mathrm{H}_{25} \mathrm{~N}_{2} \mathrm{O}$ was suggested. The exact mass of the ion was $\mathrm{m} / z, 249.1962$. The mass error was $1.2 \mathrm{ppm}(0.3 \mathrm{mDa})$ and the score was 98.61\%. Product ion spectra at CIV 10, 20 and $40 \mathrm{~V}$ revealed most abundant ions at $\mathrm{m} / \mathrm{z}, 123.0922,108.0683 \mathrm{and} 57.66975 . \mathrm{m} / \mathrm{z}$ 123.0922 is identical with protonated TDA, $m / z 108.0683$ can be traced to demethylated TDA (see Fig. 8). The most probable origin of the $\mathrm{m} / \mathrm{z} 249.1962$ ion is the reaction product of a partly hydrolyzed TDI-molecule with a fatty acid (see Fig. 10A and 10B). In the suggested substance, the isocyanate-group in position 4 (least sterically hindered) is reacted with the fatty acid. Since the uncharged molecule contains an even mass number, the nitrogen rule is obeyed.<smiles></smiles>

Fig. 10: General reaction between an isocyanate and a carboxylic acid (A) and the suggested molecule with an elemental composition of $\mathrm{C}_{15} \mathrm{H}_{24} \mathrm{~N}_{2} \mathrm{O}$ 
Fatty acids are occasionally present in the production of flexible polyurethane foams as chain-extenders. One of these acids is neo-heptanoic acid [29].

The ions that were used to identify TDA, TDA-dimers and hydroxylated TDA-dimers were missing from the collected EC fractions (MS-mode). The same pattern was observed in simultaneous UV- monitoring at $\lambda 276 \mathrm{~nm}$, as peaks 5-10 in Fig. 4 are discernible in HKC and CC samples, but not in EC samples. Peak 1 was also present, but was excluded from Fig. 11 due to its high intensity. Some of the peaks from UV monitoring correspond well with the pattern shown in Fig. 4 and have slightly shorter retention times than what was observed in MS detection, which can be explained by the DAD detector being positioned before the MS detector. The absorbance at $276 \mathrm{~nm}$ supports the presence of aromatic structures.

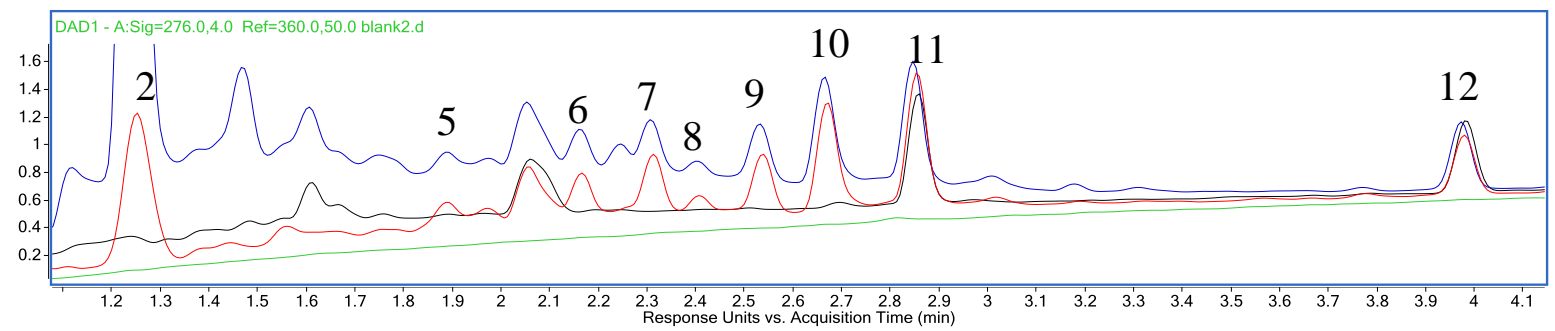

Fig. 11: UHPLC-Q-TOF results (UV-detection at $276 \mathrm{~nm}$ ) for samples collected two hours after the addition of diclofenac, which was preceded by one week of fungal immobilization on PUF (EC and HKC). Colors: EC (black), HKC (blue), CC (red) and (BL) green.

Peak 2 contains 2,4-TDA and peaks 5-10 contains TDA-dimers and monohydroxylated TDA-dimers. Peaks 3 and 4 which were tentatively identified as quinoxaline derivatives using MS and MS/MS could not be identified in CC and $\mathrm{HKC}$ at $276 \mathrm{~nm}$. The reason is probably low molar absorption coefficients for these heterocyclic compounds at this wavelength [30]. Peak 11 contains IS and peak 12 contains the suggested TDA fatty acid adduct (see Fig.10B).

The presence of the $[\mathrm{M}+\mathrm{H}]^{+}$ions corresponding to 2,4-TDA as well as the TDA-dimers and the monohydroxylated TDAdimers was monitored in EC, HKC and CC samples throughout the entire duration of the experiment. Extracted ion chromatograms (EICs) covering 2,4-TDA, the TDA dimer, and the TDA-dimer-OH at retention times of 1.3, 2.2 and 2.7 minutes, respectively, were integrated to compare levels between the three different experimental conditions over the course of the experiment.

The ratios of the integrated EICs of 2,4-TDA $(\mathrm{m} / z$ 123.0919), the TDA-dimer $(\mathrm{m} / z$ 271.1562), the TDA-dimer-OH $(\mathrm{m} / z$ 287.1509) and diclofenac d-4 (m/z 300.0496) in CC and HKC are shown in Fig. 12. The ions at $m / z, 123.0919, m / z, 271.1562$ and $\mathrm{m} / \mathrm{z} 287.1509$ were not detected in EC samples.

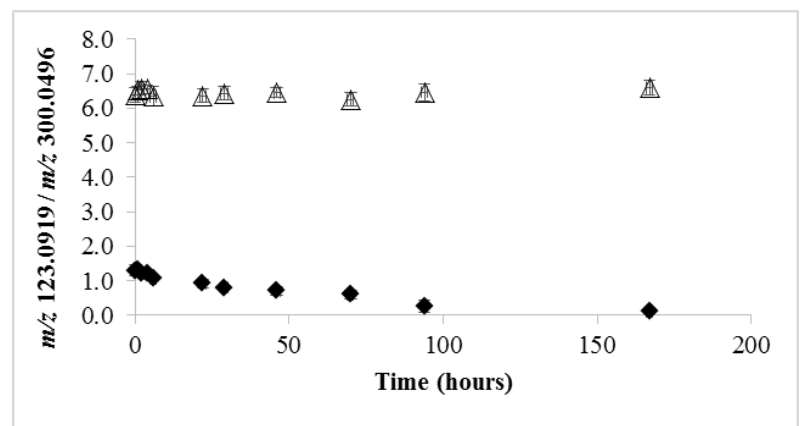

(A)

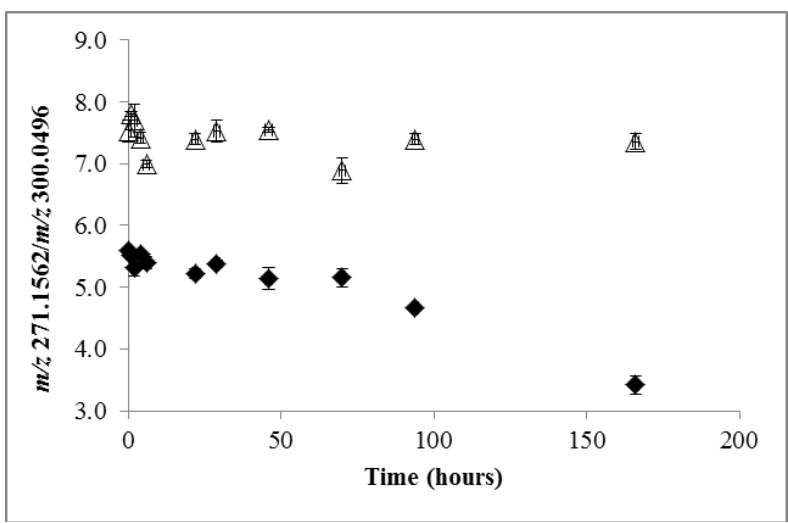

(B) 


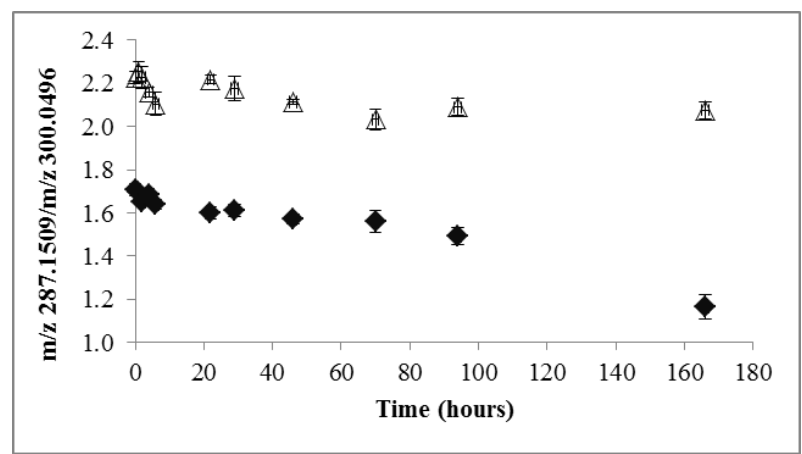

(C)

Fig.12: A representation of ratios of integrated extracted ion chromatograms (EIC) of CC $(\Delta)$ and HKC ( $\bullet)$ samples for $(\mathrm{A})$ 2,4TDA $\left(\mathrm{m} / z, 123.0919, t_{\mathrm{R}} 1.3 \mathrm{~min}\right)(\mathrm{B}) \mathrm{TDA}-\operatorname{dimer}\left(\mathrm{m} / \mathrm{z} 271.1562, t_{\mathrm{R}} 2.2 \mathrm{~min}\right),(\mathrm{C}) \mathrm{TDA}-\operatorname{dimer}-\mathrm{OH}\left(\mathrm{m} / z 287.1509, t_{\mathrm{R}} 2.7 \mathrm{~min}\right)$ and d4-diclofenac $\left(\mathrm{m} / z, 300.0496, t_{\mathrm{R}} 3.0 \mathrm{~min}\right)$. The graphs present the intensities of the compounds (reported as quota between 2,4TDA, TDA-dimer, TDA-dimer-OH and d4-diclofenac) throughout the duration of the experiment. Triplicate injections were performed for each sample

The initial differences in the 2,4-TDA, TDA-dimer and TDA-dimer-OH to diclofenac d-4 ratio between the CC and HKC samples (Fig. 12) can be explained by the biosorption of these substances by the dead biomass in the HKC culture and/or the influence of living fungi during the one-week immobilization period. In HKC samples, the ratio continues to decline during the entire time course of the experiment. As the fungi in these samples had been killed by autoclaving, this decline can only be attributed to biosorption by the dead biomass. The reason for the absence of the compounds in EC at the initial sampling time (shown by the absence of $\mathrm{m} / \mathrm{z}$ 123.0919, 271.1562 and 287.1509), while they were detectable in HKC can be explained by a desorption of the substances in the HKC-autoclavation procedure and eventually followed by a limited adsorption to the thermally transformed biomass. The approximate detection limit was in the low $\mathrm{nM}$ concentration range. A rapid biosorption of phenol and 2-chlorophenol has previously been reported for native and heat-killed fungal mycelia in Funalia trogii pellets [31]. Based on the results, the authors concluded that the biosorption properties of native mycelia were more effective. The difference was explained by a loss of hydrophobic moieties in the heat inactivated fungal mass. The authors also showed that for the selected adsorbates, the $\mathrm{pH}$ was of importance (optimal biosorption occurred at $\mathrm{pH} \mathrm{8).} \mathrm{The} \mathrm{functional} \mathrm{groups} \mathrm{present} \mathrm{in} \mathrm{the} \mathrm{fungal} \mathrm{mass,} \mathrm{e.g.} \mathrm{carboxyl,}$ sulfone, phosphate and amino groups, are the reason for these $\mathrm{pH}$-based differences in biosorption.

The intensity of the ion at $m / z$ 249. 1972 (suggested TDA fatty acid adduct present in peak 12 in Fig. 4) was integrated and divided by the intensity of the integrated IS-ion ( $\mathrm{m} / \mathrm{z}$ 300.0496) (see Fig. 13). The ion was initially present in EC, but was not detectable after 8 hours. No decline in HKC can be seen as for TDA, TDA-dimer and TDA-dimer-OH. The fast decline in EC can be explained by its adsorption to native biomass, bioaccumulation, biodegradation by exo- or endoenzymes and by its role as a nutrient for the fungus. A sole nutrition/biodegradation explanation is not plausible since the HKC-levels then should be declined after one week incubation. The approximately similar levels in HKC and CC show that no biosorption to dead biomass occurred.

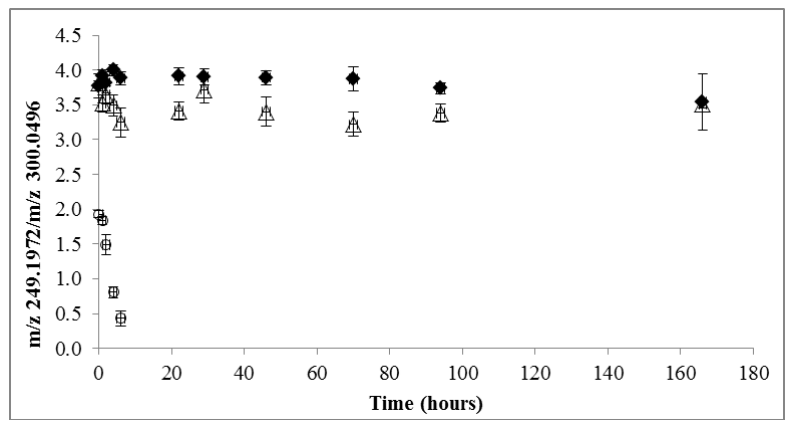

Fig. 13: A representation of ratios of integrated extracted ion chromatograms (EIC) of CC $(\Delta)$ HKC ( $\downarrow)$ and EC (o) samples for $\mathrm{m} / \mathrm{z}$ 249.1972, $\left.t_{\mathrm{R}} 4.0 \mathrm{~min}\right)$ and d4-diclofenac $\left(\mathrm{m} / \mathrm{z} 300.0496, t_{\mathrm{R}} 3.0 \mathrm{~min}\right)$. The graph presents the intensities of the compounds (reported as a quota between $\mathrm{m} / \mathrm{z} 249.1972$ and $\mathrm{d} 4$-diclofenac) throughout the duration of the experiment. Triplicate injections were performed for each sample,

In the present study, the concentration of 2,4-TDA was approximated to be considerably higher than what was expected based on results from a previous study in which 2,4-TDI was extracted from PUF using toluene. Gagné et al. [5] reported a mean 2,4TDI content of $5 \mathrm{ng} \mathrm{g}^{-1}$ in three different PUF samples. A similar content of 2,4-TDA in the PUF cubes that were used in the present investigation would translate to a $0.3 \mathrm{nM}$ concentration. However, the results depicted in Figures 12 indicate that the concentrations of 2,4-TDA and its derivatives were present at similar levels as the $0.1 \mathrm{mg} \mathrm{L}^{-1}$ diclofenac-d4 internal standard (0.3 
$\mu \mathrm{M})$, although a direct comparison is impossible due to differences in how readily TDA-containing compounds and diclofenac ionize.

The high and persistent levels of 2,4-TDA and its derivatives in CC can be explained by the low adsorption of these compounds to PUF. It is known that molecules containing aromatic structures are easily adsorbed by PUF through hydrophobic interactions [22, 32-34]. However, in the present investigation, which was conducted at a $\mathrm{pH}$ of 4, the amines are mainly protonated. Only limited electrostatic interactions between the adsorbent and adsorbate are supposed to occur since the urethane (carbamate) bonds are not considered to act neither as acids or nor as bases over the normal pH range (1-14) [35]. However, there are reports that claim that these bonds are positively charged at low pHs [36,37] which would lead to an electrostatic repulsion of the protonated TDA molecules. The use of amine-based polymers to attract negatively charged molecules at acidic pHs has been previously reported [38].

No traces of TDA or its dimer-forms could be detected at nM concentration levels from the collected EC fractions. There are four hypotheses that could explain this lack of these compounds in EC:

1. Biosorption to the T. versicolor mycelial cell walls

2. Bioaccumulation in T. versicolor (build-up of compounds within mycelial cells that excludes metabolism)

3. T. versicolor uses the target analytes as a nutrient supply, including endo-enzymatic catalysed catabolism and metabolism

4. Biodegradation of the target analytes by exoenzymes secreted from the fungus (extracellular catabolism)

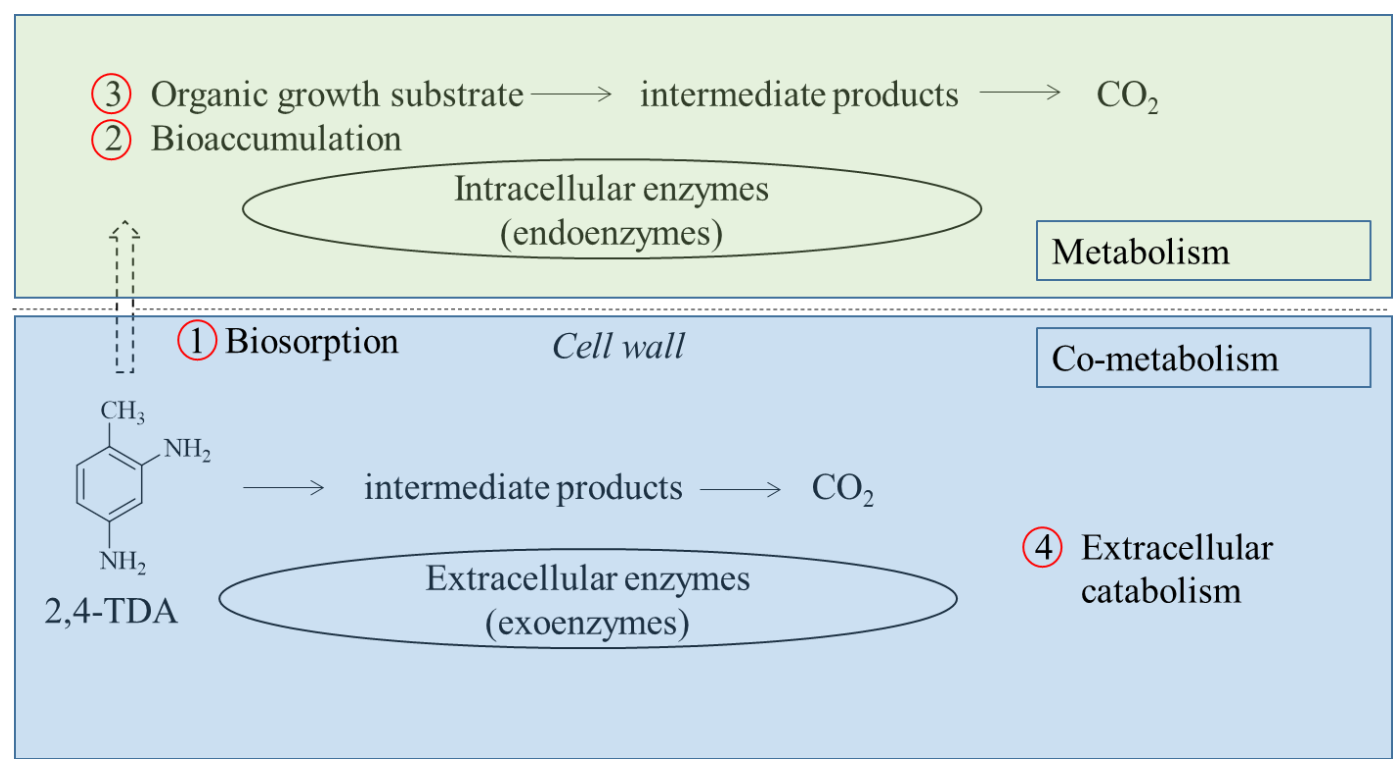

Fig. 14: Different theories for the decline of 2,4-TDA in biodegradation experiments using T. versicolor. Intracellular processes are illustrated in green and extracellular processes in blue. Co-metabolism is the transformation of a non-growth substance in the presence of a growth substrate.

Extracellular biodegradation is often mentioned as the primary way that fungi contribute to biodegradation. In this process, the fungus requires an adequate supply of nutrients to secrete exoenzymes. In T.versicolor, there are at least four non-specific exoenzymes that are present (laccase, manganese peroxidase (MnP), lignin peroxidase (LiP) and versatile peroxidase (VP)). In a previous study, an increased laccase activity was monitored during the time course [22]. However, the other three degradation principles must also be considered. Of special interest is the possibility that the fungus can use TDA and its derivatives as nutrients. In the presented experiments, nitrogen and carbon, both of which are essential for fungal growth, were supplied as glucose (carbon source) and ammonium tartrate (carbon and nitrogen source). TDA and its derivatives are small molecules that contain both carbon and nitrogen. It cannot be excluded that they penetrated through the fungal cell walls and lipid bilayers through passive transport dependent on concentration gradients (i.e. not active, energy-driven transport). It has previously been reported that $T$. versicolor can use phenol as the sole carbon source [39]. Furthermore, it has been reported that the saprophytic fungal species Rhizopus sp. and Fusarium sp. can use aniline as the sole source of both carbon and nitrogen [40]. Similarly, among mycorrhizal fungi, it has been shown that the ericoid endophyte Hymenoscyphus ericae can utilize fatty acids as efficient as glucose [41].

\section{CONCLUSIONS}

The presented experiments have demonstrated the ease by which it is possible to extract residual TDA and its derivatives from PUF in an acidic environment. Furthermore, it has been shown that PUF-immobilized Trametes versicolor is able to remove these compounds. It cannot be excluded that the fungus at least partly used the TDA compounds as nutrients (i.e. carbon and nitrogen source). The results are important to medical applications that require PUF that is free of residual TDA. If not already 
performed, an initial acidic extraction of PUF by which these TDA-derivatives are removed would minimize the risk of an unwanted future leakage. The subsequent removal of these substances from the extracts using immobilized T. versicolor, as described in the present study, can be of interest as a waste treatment procedure. Future experiments aiming to investigate the capability of $T$. versicolor to use compounds like TDA as a nutrient source must include careful calculations about the nutrient supply necessary to keep the fungus viable during experiments.

\section{ACKNOWLEDGEMENTS}

The authors would like to extend thanks to Maria Ahlgren, M.Sc., and Linda Persson, M.Sc., at GE Healthcare Bio-Sciences AB, Uppsala, Sweden, for their skillful technical assistance.

The authors are also grateful to Ivana Eichlerová, PhD, at the Institute of Microbiology, Academy of Sciences of the Czech Republic in Prague for supplying T. versicolor. This research did not receive any specific grant from funding agencies in the public, commercial, or not-for-profit sectors.

\section{References}

\section{REFERENCES}

[1] Ionescu, M. (2005). Relationships between structure and polyurethane properties: Chemistry and Technology of Polyols for Polyurethanes. Shropshire, Rapra Technology Limited, United Kingdom.

[2] Pavlova, M., Draganova, M. \& Novakov, P. (1985). Hydrolytic stability and protective properties of polyurethane oligomers based on polyester/ether/polyols. Polymer 26: 1901-1905. DOI: $\underline{10.1016 / 0032-3861(85) 90023-0}$

[3] Daka, J.N. \& Chawl, A.S. (1993). Release of chemicals from polyurethane foam in the Meme breast implant. Biomaterials Artificial Cells and Immobilization Biotechnology 21(1): 23-46. DOI:10.3109/10731199309118294

[4] Niyogi, D.R. \& Gandhi, K.S. (1999). Water blown free rise polyurethane foams. Polymer Engineering \&Science 39(1): 199209. DOI: $10.1002 /$ pen. 11408

[5] Gagné, S., Lesage, J., Ostiguy, C. \& Tra, H.V. (2003). Determination of unreacted 2,4-toluene diisocyanate (2,4TDI) and 2,6-toluene diisocyanate (2,6TDI) in foams at ultratrace level by using HPLC-CIS-MS-MS.Analyst 128(12): 1447-1451. DOI: $10.1039 / \mathrm{B} 310463 \mathrm{j}$

[6] Krone, C.A., Ely, J.T.A., Klingner, T. \& Rando,R.J. (2003). Isocyanates in flexible polyurethanefoams. Bull Environmental Contamination and Toxicology 70: 328-335. DOI: 10.1007/s00128-002-0195-2

[7] Arnold, S.M., Collins, M.A., Graham, C., Jolly, A.T., Parod, R.J., Poole, A., Schupp T., Shiotsuka, R.N. \& Woolhiser, M.R. (2012). Risk assessment for consumer exposure to toluene diisocyanate (TDI) derived from polyurethane flexible foam. Regulatory Toxicology and Pharmacology 64: 504-515. DOI:10.1016/j.yrtph.2012.07.006

[8] Shanmugam, K., Subrahmanyam, S., Tarakad, S.V., Kodandapani, N. \& Stanly, D.F. (2001). 2,4- toluene diamines-their carcinogenity, biodegradation, analytical techniques and an approach towards development of biosensors. Analytical Sciences 17: 1369-1374. DOI: $10.2116 /$ analsci.17.1369

[9] Cheung, Y.L., Snelling, J., Mohammed, N.N.D., Gray, T.J.B. \& Ioannides, C. (1996). Interaction with the aromatic hydrocarbon receptor, CYP1A induction, and mutagenicity of a series of diaminotoluenes: Implications for their carcinogenicity. Toxicology and Applied Pharmacology 139: 203-211. DOI: 10.1006/taap.1996.0159

[10] Hester Jr. T.R., Ford, N.F., Gale, P.J., Hammett, J.L., Raymond, R., Turnbull, D., Phil,D., Frankos, V.H. \& Cohen, M.B. (1997). Measurements of 2,4-toluenediamine in urine and serum samples from women with Même or Replicon breast implants. Plastic and Reconstructive Surgery 100(5): 1291-1298.

[11] Luu, H.M.D., Hutter, J.C. \& Bushar, H.F. (1998). A physiologically based pharmacokinetic model for 2,4- toluenediamine leached from polyurethane foam-covered breast implants. Environmental Health Perspectives 106(7): 393-400. DOI: 10.1289/ehp.106-1533474

[12] Cauich-Rodriguez, J.V., Chan-Chan, L.H., Hernandez-Sanchez, F. \& Cervantes-Uc, J.M. (2013).Degradation of polyurethanes for cardiovascular applications. Advances in Biomaterials Science and Biomedical Applications DOI: $\underline{10.5772 / 53681}$ [accessed on October 7, 2017].

[13] Johnson, J.R., Karlsson, D., Dalene, M. \&. Skarping, G. ( 2010). Determination of aromatic amines in aqueous extracts of polyurethane foam using hydrophilic interaction liquid chromatography and mass spectrometry. Analytica Chimica Acta 678: 117-123. DOI: 10.1016/j.aca.2010.08.020 
[14] Physico-chemical property predictors, ChemAxon, Software for Chemistry and Biology. https://www.chemaxon.com/products/calculator-plugins/prperty-predictors/\#pka [accessed on October 7, 2017].

[15] Mutsuga, M., Yamaguchi, M. \& Kawamura, Y. (2014). Quantification of isocyanates and amines in polyurethane foams and coated products by liquid chromatography-tandem mass spectrometry. Food Science \& Nutrition, 2: 156-163. DOI: $10.1002 /$ fsn 3.88

[16] Hall, K., van Pyrus, F.A.L. \& Young, R.J. (2001). Toluenediamine in polyurethane foams, hazard or artefact. Plastics, Rubber and Composites 30(9): 426-433. DOI: 10.1179/146580101101541831

[17] Ketata, N., Sanglar, C., Waton, H., Alamercery, S., Delolme, F., Raffin, G. \& Grenier- Loustalot, M.F.(2004). Thermal degradation of polyurethane bicomponent systems in controlled atmospheres. Polymers and Polymer Composites 13(1): 1-26. DOI: $10.1177 / 096739110401200801$

[18] Hosseini, J. \& Shokri, A. (2017). Employing UV/ $\mathrm{H}_{2} \mathrm{O}_{2}$ process for degradation of 2,4-diaminotoluene in synthetic wastewater. Archives on Hygiene Sciences 6(2): 121-127.

[19] Asakura, S.\& Okazaki, S. (1995). Biodegradation of toluene diamine (TDA) in activated sludge acclimated with aniline and TDA. Chemosphere 30(11): 2209-2217. DOI: 10.1016/0045-6535(95)00093-N

[20] Kim, M.N., Jang, J.C., Lee, I.M. \& Yoon, J.S. (2002). Toxicity and biodegradation of diamines. Journal of Environmental Science and Health, Part B 37: 53-64. DOI: 10.1081/PFC-120002897

[21] Yemendzhiev, H., Terziyska, A., Manasiev, J. \& Alexieva, Z (2011). Degradation of mixed aromatic pollutants by Trametes versicolor strain 1, Biotechnology and Biotechnological Equipment 25(4), Suppl,39-40. DOI: 10.5504/BBEQ.2011.0119

[22] Stenholm, A., Hedeland, M., Arvidsson, T. \& Pettersson, C.E. (2018). Removal of diclofenac from a non-sterile aqueous system using Trametes versicolor with an emphasis on adsorption and biodegradation mechanisms. Environmental Technology DOI: $10.1080 / 09593330.2018 .1444098$

[23] ChemSpider, http://www.chemspider.com/Chemical-Structure.1906.html

[24] ChemCalc: a building block for tomorrow's chemical infra-structures. Padiny, L.\& Borel, A. Journal of Chemical Information and Modeling. DOI: 10.1021/ci300563h

[25] Kirss, V. \& Park, J,C. (1969). Purification of toluenediamine mixtures by vacuum distillation. U.S. Patent 3.420,752.

[26] Rasal, K.B. \& Yadav, G.D. ( 2016). Carbon dioxide mediated novel synthesis of quinazoline-2,4 (1H,3H)-dione in water. Organic Process Research \& Development 20: 2067-2073. DOI: 10.1021/acs.oprd.6b00244

[27] Pereira, J.A., Pessoa, A.M., Cordeiro, M.N.D.S., Fernandes, R., Prudêncio ,C., Noronha, J.P.\& Vieira, M. (2015). Quinoxaline, its derivatives and applications: A state of the art review. European Journal of Medicinal Chemistry 97: 664-672. DOI: 10.1016/j.ejmech.2014.06.058

[28] Ábraham, J., Magyari, M. \& Lakatos, J. (2015). Formation of tars in the TDA-TDI synthesis and experimental study of its removal in the TDI recovery processes. Materials Science and Engineering 40(1): 5-16.

[29] Burdeniuc, J.J, Tobias J,D, Jeon, Y.M. \& Keller, R.J. (2011). Process for producing flexible polyurethane foam. European Patent 2591031 B1.

[30] Abdulgane, H.A., Kamel, B.A.F., Askar, F.W. \& Mohammed, H.H. (2018). Synthesis and theoretical study of new quinazolinone derivatives containing various heterocyclic moieties. International Research Journal of Pharmacy 9(5): 75-80. DOI: $10.7897 / 2230-8407.09576$

[31] Bayramoglu, G., Gursel, I., Tunali, Y. \& Arica, M.Y. (2009). Biosorption of phenol and 2- chlorophenol by Funalia trogii pellets. Bioresource Technology 100: 2685-2691. DOI: 10.1016/j.biortech.2008.12.042

[32] Enkiri, F., Hulen, C. \& Legault-Demare, J. (1995). Hydrophobic adsorption of aromatic compounds on polyurethane foam as carbon source for Pseudomonas growth. Applied Microbiology and Biotechnology 44: 539-545. DOI: 10.1007/BF00169957 
[33] El-Shahawi, M.S., Aldhaheri, S.M. (1996). Preconcentration and separation of acaricides by polyether based polyurethane foam. Analytica Chimica Acta 320: 277-287. DOI: 10.1016/0003-2670(95)00450-5

[34] Schumack, L. \& Chow. A. (1987). Extraction of aromatic organic compounds by polyurethane foam. Talanta 34(11): 957962. DOI: $10.1016 / 0039-9140(87) 80136-4$

[35] de Ruiter, J. Principles of Drug Action 1, Spring (2005). Amides and related functional groups. http://www.auburn.edu/ deruija/pda1_amides.pdf [accessed on September 20, 2018].

[36] Capella-Peiró, M.E., Bose, D., Durgbanshi, A., Gil-Augusti, M., Carda-Broch, S. \& Esteve-Romero, J.(2004). Determination of carbamate pesticides using micellar liquid Chromatography. Indian Journal of Chemistry 42A: 1417-1422.

[37] Alfassi, Z. \& Wai, C.M. (1991). Preconcentration techniques for trace elements: Separation and preconcentration of trace elements and inorganic species on solid polyurethane foam sorbents, first edition., Boca Raton, USA.

[38] Terangpi, P. \& Chakraborty, S. (2016). Adsorption kinetics and equilibrium studies for removal of acid azo dyes by aniline formaldehyde condensate. Applied Water Science 7(7): 3661-3671.

[39] Yemendzhiev, H., Gerginova, M., Krastanov, A., Stoilova, I. \& Alexieva, Z. (2008). Growth of Trametes versicolor on phenol. Journal of Industrial Microbiology and Biotechnology. 35: 1309-1312. DOI: 10.1007/s10295-008-0412-z

[40] Emtiazi, G., Satarii, M. \& Mazaherion, F. (2001). The utilization of aniline, chlorinated aniline, and aniline blue as the only source of nitrogen by fungi in water. Water Research, 35(5): 1219-1224. DOI: 10.1016/S0043-1354(00)00370-5

[41] Bajwa, R. \& Read, D.J. (1989). Responses of the ericoid endophyte Hymenoscyphus ericae and of mycorrhizal and nonmycorrhizal seedlings of Vaccinium to fatty and phenolic acids. Science International (Lahore) 1(6): 384-387. 